\title{
Alzheimer's Disease Prognosis is Captured by a Down-Upsized Incidence Poisson Distribution
}

\author{
Ramalingam Shanmugam \\ School of Health Administration, Texas State University, San Marcos, TX 78666, USA
}

Received 2013-07-02, Revised 2013-07-31; Accepted 2013-07-22

\begin{abstract}
Alzeimer disease is a daunting nightmare to both the medical and families of the patients. Tireless efforts are made by the medical researchers to treat Alzheimer's patients. The Alzhiemer patients' data provides clues about its prognosis and they can be identifies when the data are analyzed and understood correctly. The data analysis is a first step in the path to breakthrough treatment. Towards this aim, this article introduces a new statistical distribution and calls it Down-Upsized Incidence Poisson Distribution (DUIPD). The properties of DUIPD are obtained and illustrated to interpret the patterns in the prognosis of two group of Alzheimer's patients. In one group, the patients received a doseage of lecithin and other in the control group received placebo drug.
\end{abstract}

Keywords: Likelihood Ratio, Poisson Distribution, Power, p-Value, Count Model, Survival Function

\section{INTRODUCTION}

What is Alzheimer's disease? This disease was named after a German psychiatrist, Alois Alzheimer who first discovered it in 1908. Moore and Moore (2012) provides a historical account, research centers and treatment facilities of Alzheimer's disease. There were notable men and women who had this disease. When and where the Alzheimer's disease occurs is narrated in Braak and Tredici (2012). The past U.S. president Ronald Reagon had this disease until his death. The former prime minister of England, Mr. Harold Wilson had this disease. It is a brain disease causing severe memory impairment. Its cause still remains unknown. Hence, there is no preventive or effective treatment as of now. It occurs with senile plagues, neurofibrillary tangles, dystrophic neuritis and neuronal loss. Medical community feels that excessive protein of $\beta$ Amylpod or a genetic mutation affecting Amyloid Precursor Protein (APP) genes. Perhaps, about 100,000 cases existed in the world in 2004. The average life expectancy is only seven years once the disease is onset, though it commonly occurs among those aged 65 or older.

A measure to capture the deterioration or improvement is the number of words an Alzheimer's patient can remember from a list s/he read before. While such an assessment is periodically made, the medical team treating the Alzheimer's patient desires to understand and explain the patient's disease prognosis with an ultimate purpose to continue on or alter the treatment. None among the current distribution in the literature is equipped to bring forth the prognosis of the disease. Hence, there is a need to invent a new probability pattern and it is done first. This new probability pattern is named Down-Upsized Incidence Poisson Distribution (DUIPD). The statistical properties of DUIPD are identified and then applied to the data in Table 1 and 2 to understand the prognosis of the disease in 48 Alzheimer's patients (26 are from the control group receiving placebo and 22 are from a treatment group receiving lecithin). The data are cited in Everitt and Pickler (2004) after a description of the randomoization in the clinical trial. The data entry in the table is $\mathrm{Y}$, the number of words remembered from a list seen earlier. The variables Y0, Y1, Y2, Y4 and Y6, in the Table 1 and 2 , denote respectively the number of words the patient remembered in the beginning, one, two, four and six months. The results are interpreted. A few conclusive thought are stated in the end for future research directions. 
Ramalingam Shanmugam / American Medical Journal 4 (2): 150-159, 2013

Table 1. Number of words remembered by the Alzhiemner's patients in the control group

\begin{tabular}{lrrrrrlllll}
\hline Patient & Y0 & Y1 & Y2 & Y4 & Y6 & $1+$ effect1 & $1+$ effect2 & $1+$ effect3 & 1+effect4 & Judgement \\
\hline 1 & 20 & 19 & 20 & 20 & 18 & 0.975 & 0.98333 & 0.9875 & 0.97 & No Improvement \\
2 & 14 & 15 & 16 & 9 & 6 & 0.725 & 0.75000 & 0.6750 & 0.60 & No Improvement \\
3 & 7 & 5 & 8 & 8 & 5 & 0.300 & 0.33333 & 0.3500 & 0.33 & No Improvement \\
4 & 6 & 10 & 9 & 10 & 10 & 0.400 & 0.41667 & 0.4375 & 0.45 & No Improvement \\
5 & 9 & 7 & 9 & 5 & 6 & 0.400 & 0.41667 & 0.3750 & 0.36 & No Improvement \\
6 & 9 & 10 & 9 & 11 & 11 & 0.475 & 0.46667 & 0.4875 & 0.50 & No Improvement \\
7 & 7 & 3 & 7 & 6 & 3 & 0.250 & 0.28333 & 0.2875 & 0.26 & No Improvement \\
8 & 18 & 20 & 20 & 23 & 21 & 0.950 & 0.96667 & 1.0125 & 1.02 & 2ndHalfImprovement \\
9 & 6 & 10 & 10 & 13 & 14 & 0.400 & 0.43333 & 0.4875 & 0.53 & No Improvement \\
10 & 10 & 15 & 15 & 15 & 14 & 0.625 & 0.66667 & 0.6875 & 0.69 & No Improvement \\
11 & 5 & 8 & 7 & 3 & 12 & 0.325 & 0.33333 & 0.2875 & 0.35 & No Improvement \\
12 & 11 & 11 & 8 & 10 & 9 & 0.550 & 0.50000 & 0.5000 & 0.49 & No Improvement \\
13 & 10 & 2 & 9 & 3 & 2 & 0.300 & 0.35000 & 0.3000 & 0.26 & No Improvement \\
14 & 17 & 12 & 14 & 15 & 13 & 0.725 & 0.71667 & 0.7250 & 0.71 & No Improvement \\
15 & 16 & 15 & 13 & 7 & 9 & 0.775 & 0.73333 & 0.6375 & 0.60 & No Improvement \\
16 & 7 & 10 & 4 & 10 & 5 & 0.425 & 0.35000 & 0.3875 & 0.36 & No Improvement \\
17 & 5 & 0 & 5 & 0 & 0 & 0.125 & 0.16667 & 0.1250 & 0.10 & No Improvement \\
18 & 16 & 7 & 7 & 6 & 10 & 0.575 & 0.50000 & 0.4500 & 0.46 & No Improvement \\
19 & 5 & 6 & 9 & 5 & 6 & 0.275 & 0.33333 & 0.3125 & 0.31 & No Improvement \\
20 & 2 & 1 & 1 & 2 & 2 & 0.075 & 0.06667 & 0.0750 & 0.08 & No Improvement \\
21 & 7 & 11 & 7 & 5 & 11 & 0.450 & 0.41667 & 0.3750 & 0.41 & No Improvement \\
22 & 9 & 10 & 17 & 10 & 6 & 0.475 & 0.60000 & 0.5750 & 0.52 & No Improvement \\
23 & 2 & 5 & 6 & 7 & 6 & 0.175 & 0.21667 & 0.2500 & 0.26 & No Improvement \\
24 & 7 & 3 & 5 & 5 & 5 & 0.250 & 0.25000 & 0.2500 & 0.25 & No Improvement \\
25 & 10 & 13 & 19 & 17 & 17 & 0.575 & 0.70000 & 0.7375 & 0.76 & No Improvement \\
26 & 7 & 5 & 8 & 8 & 6 & 0.300 & 0.33333 & 0.3500 & 0.34 & No Improvement \\
\hline & & & & & & & & &
\end{tabular}

Table 2. Number of words remembered by the Alzhiemner's patients in the treatment group

\begin{tabular}{lrrrrrlllll}
\hline Patient & Y0 & Y1 & \multicolumn{1}{rl}{ Y2 } & \multicolumn{1}{c}{ Y4 } & Y6 & $1+$ effect1 & $1+$ effect2 & $1+$ effect3 & $1+$ effect4 & Judgement \\
\hline 1 & 9 & 11 & 14 & 11 & 14 & 1.11111 & 1.25926 & 1.25000 & 1.31111 & Good Improvement \\
2 & 6 & 7 & 9 & 12 & 16 & 0.72222 & 0.81481 & 0.94444 & 1.11111 & End Improvement \\
3 & 13 & 18 & 14 & 20 & 14 & 1.72222 & 1.66667 & 1.80556 & 1.75556 & Good Improvement \\
4 & 9 & 10 & 9 & 8 & 7 & 1.05556 & 1.03704 & 1.00000 & 0.95556 & Good Improvement \\
5 & 6 & 7 & 4 & 5 & 4 & 0.72222 & 0.62963 & 0.61111 & 0.57778 & No Improvement \\
6 & 11 & 11 & 5 & 10 & 12 & 1.22222 & 1.00000 & 1.02778 & 1.08889 & Good Improvement \\
7 & 7 & 10 & 11 & 8 & 5 & 0.94444 & 1.03704 & 1.00000 & 0.91111 & Good Improvement \\
8 & 8 & 18 & 10 & 15 & 14 & 1.44444 & 1.33333 & 1.41667 & 1.44444 & Good Improvement \\
9 & 3 & 3 & 3 & 1 & 3 & 0.33333 & 0.33333 & 0.27778 & 0.28889 & No Improvement \\
10 & 4 & 10 & 9 & 17 & 10 & 0.77778 & 0.85185 & 1.11111 & 1.11111 & 2 ndHalfImprovement \\
11 & 11 & 10 & 5 & 15 & 10 & 1.16667 & 0.96296 & 1.13889 & 1.13333 & Overall Improvement \\
12 & 1 & 3 & 2 & 2 & 5 & 0.22222 & 0.22222 & 0.22222 & 0.28889 & No Improvement \\
13 & 6 & 7 & 7 & 6 & 7 & 0.72222 & 0.74074 & 0.72222 & 0.73333 & No Improvement \\
14 & 0 & 3 & 2 & 0 & 0 & 0.16667 & 0.18519 & 0.13889 & 0.11111 & No Improvement \\
15 & 18 & 19 & 15 & 17 & 20 & 2.05556 & 1.92593 & 1.91667 & 1.97778 & Good Improvement \\
16 & 15 & 15 & 15 & 14 & 12 & 1.66667 & 1.66667 & 1.63889 & 1.57778 & Good Improvement \\
17 & 14 & 11 & 8 & 10 & 8 & 1.38889 & 1.22222 & 1.19444 & 1.13333 & Good Improvement \\
18 & 6 & 6 & 5 & 5 & 8 & 0.66667 & 0.62963 & 0.61111 & 0.66667 & No Improvement \\
19 & 10 & 10 & 6 & 10 & 9 & 1.11111 & 0.96296 & 1.00000 & 1.00000 & Overall improvement \\
20 & 4 & 6 & 6 & 4 & 2 & 0.55556 & 0.59259 & 0.55556 & 0.48889 & No Improvement \\
21 & 4 & 13 & 9 & 8 & 7 & 0.94444 & 0.96296 & 0.94444 & 0.91111 & No Improvement \\
22 & 14 & 7 & 8 & 10 & 6 & 1.16667 & 1.07407 & 1.08333 & 1.00000 & Good Improvement \\
\hline
\end{tabular}


Ramalingam Shanmugam / American Medical Journal 4 (2): 150-159, 2013

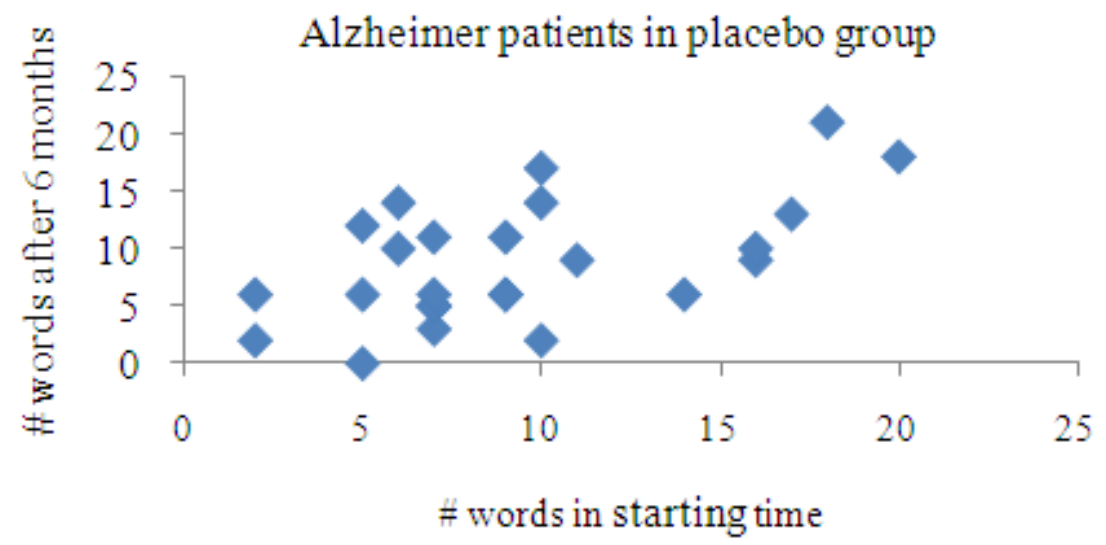

Fig. 1. Pattern in control group

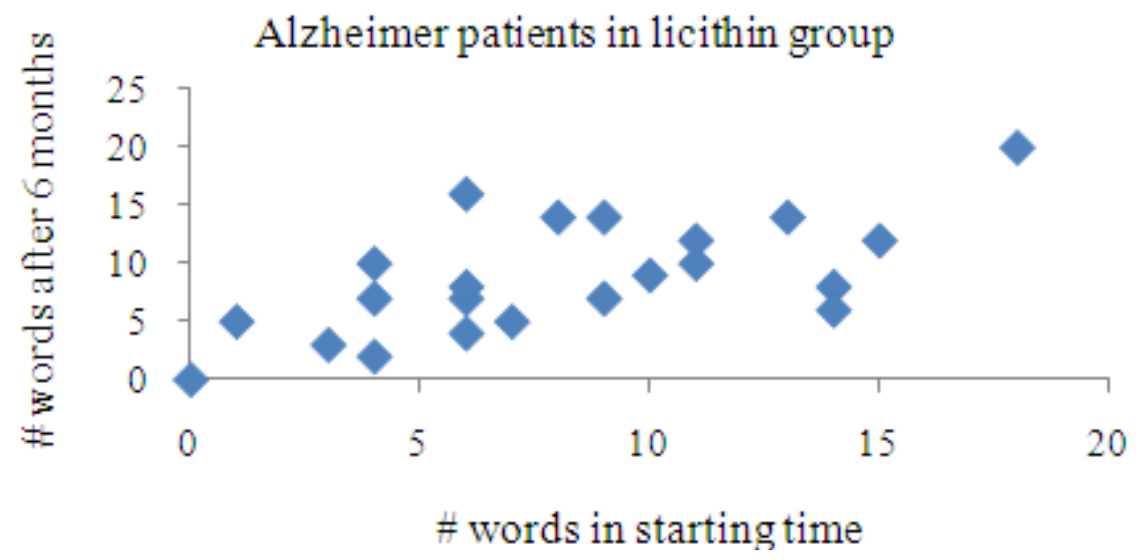

Fig. 2. Pattern in the treatment group

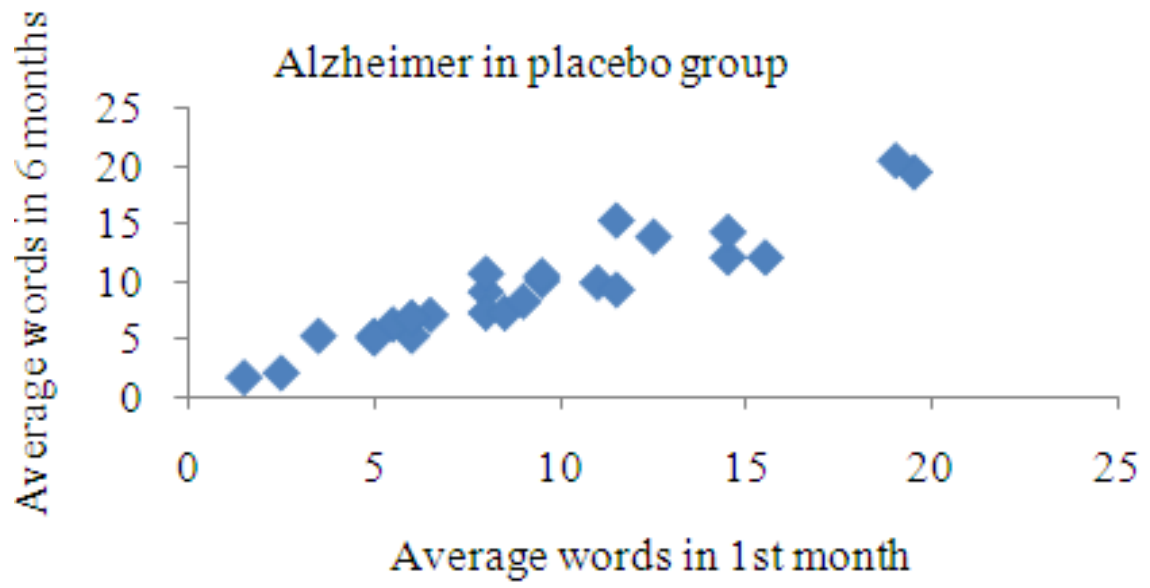

Fig. 3. Moving averages in control group 


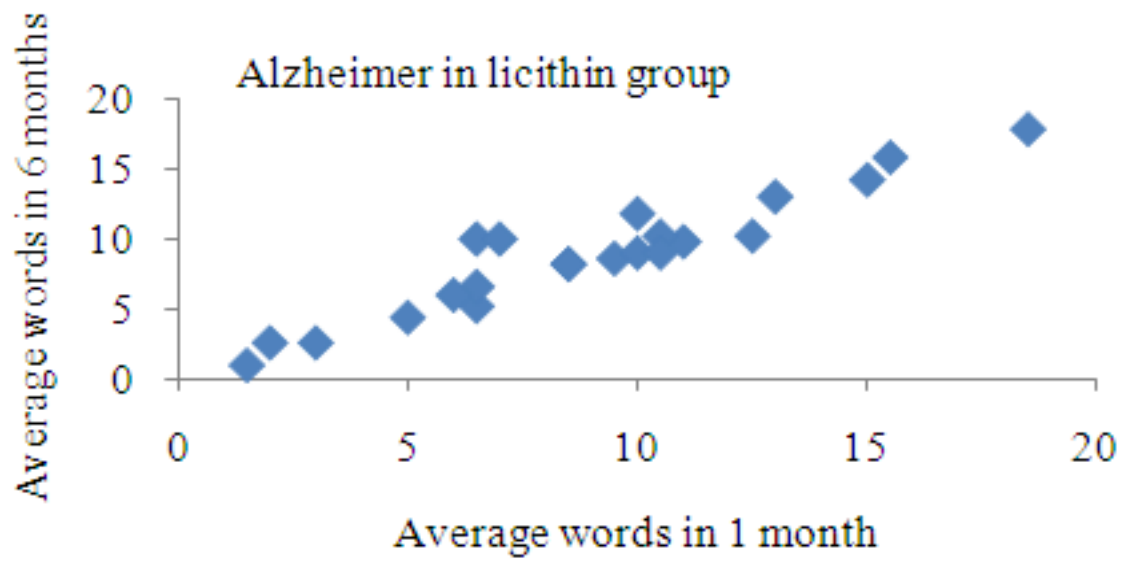

Fig. 4. Moving averages in treatment group

\subsection{Comparative Patterns}

First, we need to catch the similarities and differences in the data pattern of the control versus treatment groups. See the data configurations in Fig. 1 and 2 for the control and in Fig. 3 and 4 the treatment groups. The pattern for the treatment group is clearly visible compared to that for control group.

To capture the data evidence a bit more clearly, compare the Fig. 2 and 4 respectively for the control and treatment moving averages. At the end of one, two, four and six months, the average number of words remembered by each patient for the control and the treatment groups are computed and are displayed by the variables Ave1, Ave2, Ave4 and Ave6 respectively. Notice that on the average, the patients in the treatment group remember more words with more consistency than how their counterparts in the control group did. One wonders the reasons for such subtle differences. These questions leads into a new line of thinking and a reformulation of the underlying model for the data analysis as it is done next.

\subsection{Down-Upsized Incidence Poisson Distribution}

Let an integer random variable, $\mathrm{B} \geq 0$ denote the number of words an Alzheimer patient can remember in the starting time with a finite incidence rate $\theta>0$. Clearly, the random variable $\mathrm{B}$ follows a Poisson probability pattern. That means Equation (1):

$$
\operatorname{Pr}(B=b \mid \theta)=e^{-\theta} \theta^{b} / b ! ; b=0,1,2 \ldots ; \theta>0
$$

Sooner the treatment begins, it will have an impact in a sense that the on the incidence rate of the patient's rememberring the words increase, remain stable or decrease depending on an unknown parameter value $\phi>1, \phi=1$ or $0<\phi<1$. The parameter $\phi>0$ is named the down-upsing effect on the incidence rate. For the medical community, the down-upsizing effect adds a valuable information to decide whether to continue with the treatment (when $\phi>1$ ) or switch to a different treatment (when $\phi<1$ ). In other words, the number of words, $\mathrm{L} \geq 0 \mathrm{~s} / \mathrm{he}$ remembers in a defined later period of time is likely to follow a Poisson probability pattern with an altered compounded parameter $\phi>0$. The total number, $\mathrm{Y}=\mathrm{B}+\mathrm{L}$ of words $\mathrm{s} / \mathrm{he}$ remember follows then a Poisson probability pattern:

$$
\begin{aligned}
& \operatorname{Pr}(\mathrm{Y}=\mathrm{y} \mid \theta, \phi)=\sum_{\mathrm{b}=0}^{\mathrm{y}} \operatorname{Pr}(\mathrm{B}=\mathrm{b} \mid \theta) \operatorname{Pr}(\mathrm{L}=\mathrm{y}-\mathrm{b} \mid \theta, \phi) \\
& =\frac{\mathrm{e}^{-\theta(1+\phi)}(\phi \theta)^{\mathrm{y}}}{\mathrm{y} !} \sum_{\mathrm{b}=0}^{\mathrm{y}}\left(\begin{array}{l}
\mathrm{y} \\
\mathrm{b}
\end{array}\right)\left(\frac{1}{\phi}\right)^{\mathrm{y}}
\end{aligned}
$$

which simplified to Equation (2):

$$
\begin{aligned}
& \operatorname{Pr}(\mathrm{Y}=\mathrm{y} \mid \theta, \phi)=\frac{\mathrm{e}^{-\theta(1+\phi)}[(1+\phi) \theta]^{\mathrm{y}}}{\mathrm{y} !} \\
& \mathrm{y}=0,1,2 . ., ; \theta>0 ; \phi>0
\end{aligned}
$$

The expression in (2) is named Down-Upsized Incidence Poisson Distribution (DUIPD). 


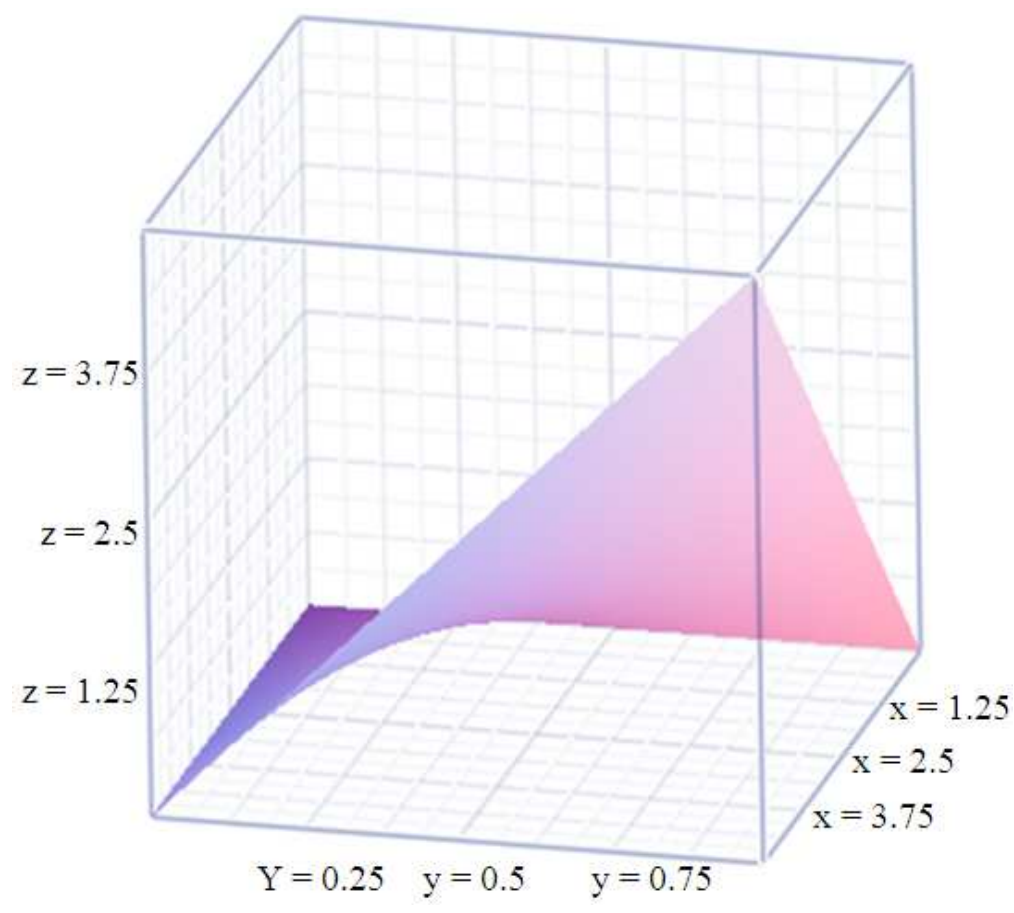

Fig. 5. The prognosis of the down-upsizing effect

When additional data on the number of words an Alzheimer's patient remembers is added to the starting data of the patient, the DUIPD should be used as the underlying model for the pooled data. In other words, with the statistical properties of (1) and (2) together, the data analysis could capture and interpret many different shifts in the prognosis of the Alzheimer's patients.

First, the mean shift is Equation (3):

$$
\mathfrak{I}_{\text {mean-shift }}=\mathrm{E}(\mathrm{Y}=\mathrm{y} \mid \theta, \phi)-\mathrm{E}(\mathrm{Y}=\mathrm{y} \mid \theta)=\phi \theta
$$

Letting $\mathrm{z}=\mathfrak{I}_{\text {mean-shift, }} \mathrm{x}=\theta$ and $\mathrm{y}=\phi$ in a three dimensional graph in Fig. 5, the prognosis of the downupsizing effect on the incidence of Alzheimer's patients would behave as in Fig. 5.

The variance shift is Equation (4):

$$
\mathfrak{J}_{\text {var-shift }}=\mathrm{E}(\mathrm{Y}=\mathrm{y} \mid \theta, \phi)-\mathrm{E}(\mathrm{Y}=\mathrm{y} \mid \theta)=\phi \theta
$$

The variance is a measure of homogeneity. It is worth noticing, by considering both (3) and (4), that the mean shift increases in the midst of increasing heterogeneity. The skewness shifts by an amount Equation (5):

$$
\mathfrak{I}_{\text {skewness-shift }}=\frac{1}{\sqrt{\theta}}\left(\frac{1}{\sqrt{1+\phi}}-1\right)
$$

Suggesting that the skewness diminishes as the down-upsizing effect increases. The kurtosis shifts by an amount Equation (6):

$\mathfrak{I}_{\text {kurtosis-shift }}=1+3(1+\phi) \theta$

Which increases with an increasing down-sizing effect. Because the Poisson probability pattern and chisquared distribution function is functionally related, the probability for an Alzheimer's patient to remember at least $\mathrm{m}$ words is then, according to 2 Equation (7):

$\operatorname{Pr}(\mathrm{Y} \geq \mathrm{m} \mid \theta, \phi)=\operatorname{Pr}\left(\chi_{\mathrm{mdf}}^{2} \leq 2(1+\phi) \theta\right)$

The probability for the Alzheimer's patient's ability to remember extra $t$ word from the list, according to (7), is then Equation (8):

$\operatorname{Pr}(\mathrm{Y} \geq \mathrm{m}+\mathrm{t} \mid \theta, \phi, \mathrm{Y} \geq \mathrm{m})=\frac{\operatorname{Pr}\left(\chi_{(\mathrm{m}+\mathrm{t}) \mathrm{df}}^{2} \leq 2(1+\phi) \theta\right)}{\operatorname{Pr}\left(\chi_{\mathrm{mdf}}^{2} \leq 2(1+\phi) \theta\right)}$ 
The shift in the Alzheimer's patient's ability due to the down-upsizing incidence effect by the treatment is then Equation (9):

$$
\begin{aligned}
& \mathfrak{J}_{\text {extra-one-word }}=\frac{\operatorname{Pr}\left(\chi_{(\mathrm{m}+1) \mathrm{df}}^{2} \leq 2(1+\phi) \theta\right)}{\operatorname{Pr}\left(\chi_{\mathrm{mdf}}^{2} \leq 2(1+\phi) \theta\right)} \\
& -\frac{\operatorname{Pr}\left(\chi_{(\mathrm{m}+1) \mathrm{df}}^{2} \leq 2 \theta\right)}{\operatorname{Pr}\left(\chi_{\mathrm{mdf}}^{2} \leq 2 \theta\right)}
\end{aligned}
$$

Having seen several properties of the new statistical distribution DUIPD in (2), it is time to check its performance with respect to actual data from a random sample of $\mathrm{n}=48$ patients as mentioned in Everitt and Pickler (2004).

With the variables $\mathrm{Y} 0$ denoting the number of words the Alzheimer's patient remembered initially before the experiment began with no down-sizing effect, the Maximum Likelihood Estimate (MLE) of the incidence parameter is $\hat{\theta}_{\text {mle }}=\mathrm{Y} 0$ and the conditional MLE of the down-upsizing effect is Equation (10.1 and 10.2):

$\hat{\theta}_{\text {mle }}=\mathrm{y} 0$

$\hat{\phi}_{\mathrm{mle}}=\left|\frac{\overline{\mathrm{y}}}{\hat{\theta}_{\mathrm{mle}}}-1\right|=\left|\frac{\overline{\mathrm{y}}}{\mathrm{y} 0}-1\right|$

where, the mean $\bar{y}$ and $\mathrm{y}^{0}$ denote respectively the overall average number and initial number of words the Alzheimer's patient has remembered. The incidence parameter's amplification factor in DUIPD (2) is $1+\hat{\phi}_{\text {mle }}$. When the amplification factor is greater than one, there is an upsizing effect and the Alzheimer's patient is considered to show improvement. When the amplification factor is lesser than one, there is a downsizing effect and the Alzheimer's patient is considered to show no improvement. When the amplification factor is just one, there exists a status quo effect and the Alzheimer's patient is considered to show no improvement or improvement.

However, of interest to a medical team might be a statistical approach to judge whether or not the upsizing effect of a patient is significant. An answer to this question has to start at a hypothesis testing of the status quo hypothesis (that is $\mathrm{H}_{0}: 1+\phi=1$ ) versus improvement hypothesis (that is $\mathrm{H}_{0}: 1+\phi>1$ ). This hypothesis testing can be done using Wald's Likelihood Ratio Principle (WLRP).
What is WLRP? It is the MLE estimated ratio $\Re_{n}$ of likelihood under the status quo over the improvement hypotheses. This is a powerful technique according to Stuart and Ord (1994). To ease the process of WLRP, consider the log-likelihood function of a random sample $\mathrm{y}_{1}, \mathrm{y}_{2}, . ., \mathrm{y}_{\mathrm{n}}$ of observations for an Alzheimer's patient in the end of $n$ time periods and it is:

$$
\ln \mathrm{L}=\text { cons } \tan \mathrm{t}-\mathrm{n} \theta(1+\phi)+n \bar{y}[\ln \theta+\ln (1+\phi)]
$$

Using the MLEs in (10.1) and (10.2), the log-WLRP is then Equation (11):

$$
-\ln \Re_{*}=\mathrm{n}\left\{\left[\overline{\mathrm{y}}\left(1+\ln \left[\frac{\mathrm{y} 0}{\overline{\mathrm{y}}}\right]+\ln \left[1+\phi^{*}\right]\right)-\mathrm{y} 0\left(1+\phi^{*}\right)\right]\right\}
$$

When $\phi=\phi$. Under the null hypothesis, the logWLRP is $-\ln \Re_{0}$ with $\phi^{*}=0$ and $-\ln \Re_{*}$ under the research hypothesis. The expression (11) follows a noncentral chi-squared distribution with non-centrality parameter $\delta_{*}=\frac{\left(\hat{\phi}_{\mathrm{MLE}}-\phi^{*}\right)}{\operatorname{var}\left(\hat{\phi}_{\mathrm{MLE}}\right)}$ where $\operatorname{var}\left(\hat{\phi}_{\mathrm{MLE}}\right)$ is a diagonal element of the variance-covariance matrix:

$$
\Sigma=\left[\begin{array}{cc}
\operatorname{var}\left(\hat{\phi}_{\mathrm{MLE}}\right) & \operatorname{cov}\left(\hat{\phi}_{\mathrm{MLE}}, \hat{\theta}_{\mathrm{MLE}}\right) \\
\operatorname{cov}\left(\hat{\phi}_{\mathrm{MLE}}, \hat{\theta}_{\mathrm{MLE}}\right) & \operatorname{var}\left(\hat{\theta}_{\mathrm{MLE}}\right)
\end{array}\right]=\mathrm{I}^{-1}
$$

which is the inverse of Fisher's information matrix:

$$
I=E\left[\begin{array}{ll}
-\partial_{\hat{\theta} \hat{\theta}}^{2} \ln L & -\partial_{\hat{\theta} \hat{\phi}}^{2} \ln L \\
-\partial_{\hat{\theta} \hat{\phi}}^{2} \ln L & -\partial_{\hat{\phi} \hat{\phi}}^{2} \ln L
\end{array}\right]
$$

After algebraic simplifications, we note that:

$$
I=n\left[\begin{array}{cc}
\frac{(1+\phi)}{\theta} & 1 \\
1 & \frac{\theta}{(1+\phi)}
\end{array}\right]
$$

Whose determinant is zero. The regular inverse is not possible because of the matrix's singularity but its generalized inverse $\mathrm{I}^{-}$of the singular matrix I is possible in the sense $\mathrm{II}^{-} \mathrm{I}=\mathrm{I}$. Bapat et al. (2013) for details about several uses of a generalized inverse. For an example, if $\mathrm{A}^{-}$is a generalized inverse matrix of the matrix ${ }^{\mathrm{A}}$, then 
$\mathrm{AA}^{-} \mathrm{A}=\mathrm{A}$ and $\mathrm{A}^{-} \mathrm{AA}^{-}=\mathrm{A}^{-}$. Such a generalized inverse for our situation is:

$$
\Sigma=\mathrm{I}^{-}=\frac{1}{\mathrm{n}}\left[\begin{array}{cc}
\frac{\theta}{(1+\phi)} & 0 \\
0 & 0
\end{array}\right]
$$

Hence, the non-centrality parameter is Equation (12):

$\delta=\frac{\mathrm{n}\left(\left[1+\hat{\phi}_{\mathrm{MLE}}\right]-\left[1+\phi^{*}\right]\right)(1+\phi)}{\theta}$

Whose MLE is Equation (13):

$$
\hat{\delta}_{*}=\frac{n\left(\frac{\bar{y}}{\mathrm{y} 0}-\left[1+\phi^{*}\right]\right) \bar{y}}{(\mathrm{y} 0)^{2}}
$$

Under the null hypothesis, the non-centrality parameter is $\hat{\delta}_{0}$ with $\phi^{*}=0$ and $\hat{\delta}_{*}$ under the research hypothesis. However, it is well known that a noncentral chi-squared distribution with a non-centrality parameter $\delta$ is approximately $\left(1+\frac{\delta}{1+\delta}\right)$ times the central chi-squared distribution with $\left(\frac{[1+\delta]^{2}}{1+2 \delta}\right)$ Degrees of Freedom (DF).

It then means that the null hypothesis $\mathrm{H}_{0}: 1+\phi=$ 1 can rejected in favor of the research hypothesis $\mathrm{H}_{0}: 1+\phi$ $>1$ when $\mathrm{n}\left\{\left[\overline{\mathrm{y}}\left(1+\ln \left[\frac{\mathrm{y} 0}{\overline{\mathrm{y}}}\right]\right)-\mathrm{y} 0\right\}\right.$ exceeds its critical value $\left(1+\frac{\hat{\delta}_{0}}{1+\hat{\delta}_{0}}\right) \chi_{\left(\frac{\left.1+\hat{\delta}_{0}\right]^{2}}{1+2 \hat{\delta}_{0}}\right) \text { DF, } \alpha}^{2}$ at a chosen significance level, $\alpha$. In other words, the p-value to reject the null in favor of the research hypothesis is Equation (14):

$$
\mathrm{p}-\text { value } \approx \operatorname{Pr}\left[\chi_{\left(\frac{\left[1+\hat{\delta}_{0}\right]^{2}}{1+2 \hat{\delta}_{0}}\right) \operatorname{DF}, \alpha}^{2}>\frac{\mathrm{n} \mid\left\{\left[\overline{\mathrm{y}}\left(1+\ln \left[\frac{\mathrm{y} 0}{\overline{\mathrm{y}}}\right]\right)-\mathrm{y} 0\right\} \mid\right.}{\left(1+\frac{\hat{\delta}_{0}}{1+\hat{\delta}_{0}}\right)}\right]
$$

The statistical power is the probability of accepting a specific research hypothesis in an event $\phi^{*}=\phi_{1} \neq 0$. That is, for a specified significance level, $\alpha$ Equation (15):

$$
\begin{aligned}
& \text { power } \approx \\
& \operatorname{Pr}\left[\chi_{\left(\frac{\left.1+\hat{\delta}_{1}\right]^{2}}{1+2 \hat{\delta}_{1}}\right) \text { DF }}^{2}<\frac{\left(1+\frac{\hat{\delta}_{1}}{1+\hat{\delta}_{1}}\right)}{\left(1+\frac{\hat{\delta}_{0}}{1+\hat{\delta}_{0}}\right)}\right. \\
& \frac{\mid\left\{\left[\overline{\mathrm{y}}\left(1+\ln \left[\frac{\mathrm{y} 0}{\overline{\mathrm{y}}}\right]\right)-\mathrm{y} 0\right\} \mid\right.}{\left\{\left[\overline{\mathrm{y}}\left(1+\ln \left[\frac{\mathrm{y} 0}{\overline{\mathrm{y}}}\right]+\ln \left[1+\phi_{1}\right]\right)-\mathrm{y} 0\left[1+\phi_{1}\right]\right\}\right.} \chi_{\left(\frac{\left(1+\hat{\delta}_{0}\right]^{2}}{1+2 \hat{\delta}_{0}}\right) \mathrm{DF}, \alpha}^{2]}
\end{aligned}
$$

\subsection{Analysis of Alzhiemer's Data in Control and Treatment Groups}

Onsider the number of words remembered from a list by a random sample of $\mathrm{n}_{1}=26$ and $\mathrm{n}_{2}=22$ Alzheimer's patients in the control and treatment group in Table 1 and 2 respectively. In the control group, the patients received placebo drug. In the reatment group, the patients received lecithin drug.

The entry in the column with the variables $\mathrm{Y} 0$ is the number of words the Alzheimer's patient remembered initially before the experiment began. According to (10.1), the Maximum Likelihood Estimate (MLE) of the incidence parameter is $\hat{\theta}_{\text {mle }}=\mathrm{Y} 0$. With the mean $\overline{\mathrm{y}}_{\text {might }}$ denoting $\quad$ Avel $=\frac{Y 0+Y 1}{2}, \quad$ Ave $2=\frac{Y 0+Y 1+Y 2}{3}$, Ave $4=\frac{\mathrm{Y} 0+\mathrm{Y} 1+\mathrm{Y} 2+\mathrm{Y} 4}{4}$ or Ave $6=\frac{\mathrm{Y} 0+\mathrm{Y} 1+\mathrm{Y} 2+\mathrm{Y} 4+\mathrm{Y} 6}{5}$ depending on whether the estimate is made at the end of one, two, fourth and sixth months respectively, the one plus down-upsizing effects are calculated using (10.2) and are shown in Table 1 and 2 for the patients. Using the expression (9), the probability for an Alzheimer's patient to remember just one-extra word in the list is computed in both groups.

The variables Y0, Y1, Y2, Y4 and Y6, in the Table 1 and 2, denote respectively the number of words the patient remembered in the beginning, one, two, four and six months. The average number of words remembered, at the end of one, two, four and six months, by each patient in the control and the treatment groups are computed and are displayed by the variables Ave1, Ave2, Ave4 and Ave6 respectively. Using (10.2), the amplifying factor was estimated at the end of one, two, four and six months, by each patient in the control and the treatment groups and they are indicated by $1+$ effect 1 , $1+$ effect $2,1+$ effect 3 and $1+$ effect 4 . When the estimate of the amplifying factor is more than one, the patient is considered to have improvement. Otherwise, the patient shows no improvement. 
Using expression (14), the p-value for the null hypothesis $\mathrm{H}_{0}: 1+\phi=1$ to be true according an Alzheimer's data in the end of 6 months is computed and displayed. Could the incidence rate have doubled? This amounts to considering $\phi^{*}=1$ in the amplifying factor. Using expression (15), the power of accepting the true hypothesis $\mathrm{H}_{1}: 1+\phi^{8}=2$ with an Alzheimer's data in the end of 6 months is computed and displayed.

Finally, we can employ the Receiver Operating Characteristic (ROC) curve to assess how well the methodology works. The ROC curve is a configuration of the confidence level (which is one minus the p-value) in terms of the power. The more convexity in the ROC means the methodology is superior.

\subsection{Alzheimer's Patients in Control Group}

The patients in the control group are received placebo drug. Scanning the estimates of the amplyfying factor in Table 1, we notice that only patient with ID \# 8 showed improvement after 2 months time. Other Alzheimer's patients in the control group showed no improvement.

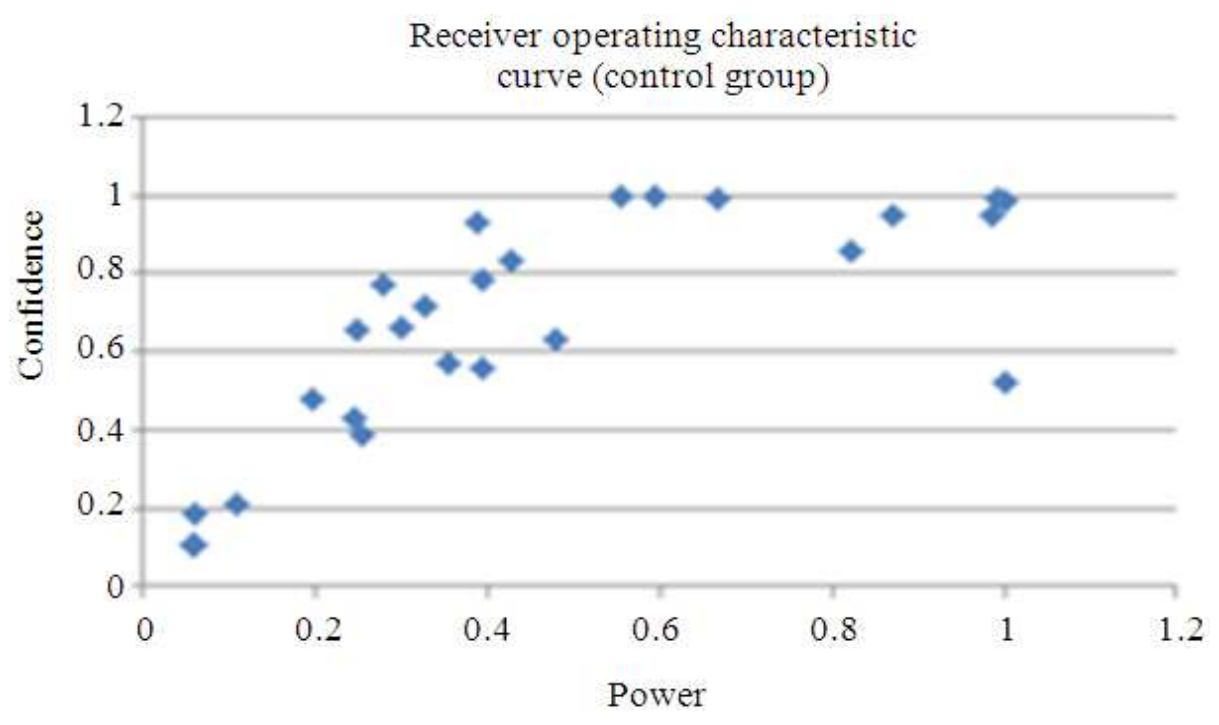

Fig. 6. ROC curve for control group

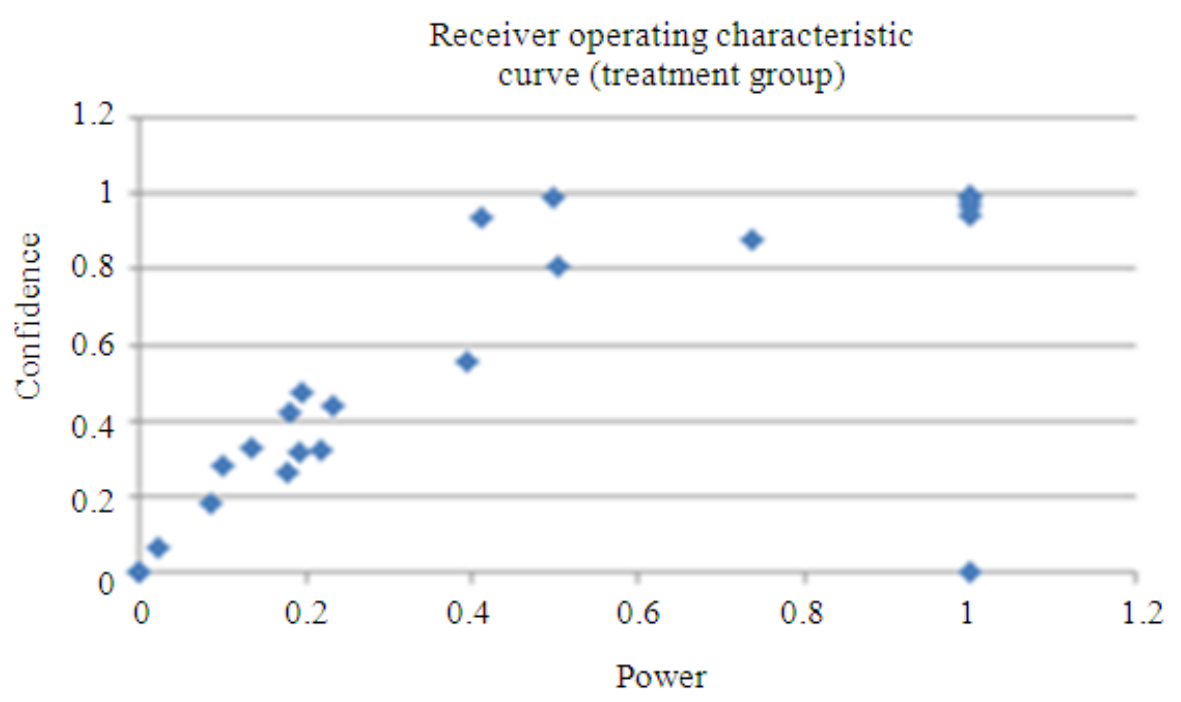

Fig. 7. ROC curve for treatment group 
Table 3. Probability to remember one more word in 1,2, 4 and 6 months in the control group

\begin{tabular}{|c|c|c|c|c|c|c|}
\hline Patient ID & $\begin{array}{l}\text { Prob to } \\
\text { remember one extra } \\
\text { word in } 1 \text { month }\end{array}$ & $\begin{array}{l}\text { Prob to } \\
\text { remember one extra } \\
\text { word in } 2 \text { months }\end{array}$ & $\begin{array}{l}\text { Prob to } \\
\text { remember one extra } \\
\text { word in } 4 \text { months }\end{array}$ & $\begin{array}{l}\text { Prob to } \\
\text { remember one extra } \\
\text { word in } 6 \text { months }\end{array}$ & $\begin{array}{l}\mathrm{p} \text {-value for } \\
\mathrm{H}_{0}: 1+\phi=1\end{array}$ & $\begin{array}{l}\text { Power with } \\
\mathrm{H}_{1}: 1+\phi=2\end{array}$ \\
\hline$\overline{1}$ & 0.56 & 0.56 & 0.56 & 0.55 & 0.82 & 0.06 \\
\hline 2 & 0.43 & 0.44 & 0.4 & 0.36 & 0.34 & 0.25 \\
\hline 3 & 0.19 & 0.21 & 0.22 & 0.21 & 0.79 & 0.11 \\
\hline 4 & 0.25 & 0.26 & 0.27 & 0.28 & $0.05 \mathrm{sig}$ & 0.99 \\
\hline 5 & 0.25 & 0.26 & 0.23 & 0.22 & 0.28 & 0.33 \\
\hline 6 & 0.29 & 0.29 & 0.3 & 0.30 & 0.57 & 0.24 \\
\hline 7 & 0.16 & 0.18 & 0.18 & 0.16 & 0.22 & 0.40 \\
\hline 8 & 0.54 & 0.55 & 0.57 & 0.58 & 0.34 & 0.30 \\
\hline 9 & 0.25 & 0.27 & 0.30 & 0.32 & $0.02 \mathrm{sig}$ & 1.00 \\
\hline 10 & 0.37 & 0.40 & 0.41 & 0.41 & $0.05 \mathrm{sig}$ & 0.87 \\
\hline 11 & 0.20 & 0.21 & 0.18 & 0.22 & 0.14 & 0.82 \\
\hline 12 & 0.33 & 0.30 & 0.30 & 0.30 & 0.52 & 0.20 \\
\hline 13 & 0.19 & 0.22 & 0.19 & 0.16 & $0.00 \mathrm{sig}$ & 0.59 \\
\hline 14 & 0.43 & 0.42 & 0.43 & 0.42 & 0.23 & 0.28 \\
\hline 15 & 0.45 & 0.43 & 0.38 & 0.36 & $0.07 \mathrm{sig}$ & 0.39 \\
\hline 16 & 0.26 & 0.22 & 0.24 & 0.22 & 0.90 & 0.06 \\
\hline 17 & 0.08 & 0.11 & 0.08 & 0.06 & $0.01 \mathrm{sig}$ & 0.67 \\
\hline 18 & 0.35 & 0.30 & 0.28 & 0.28 & $0.00 \mathrm{sig}$ & 0.55 \\
\hline 19 & 0.17 & 0.21 & 0.20 & 0.19 & 0.37 & 0.48 \\
\hline 20 & 0.05 & 0.04 & 0.05 & 0.05 & 0.61 & 0.26 \\
\hline 21 & 0.28 & 0.26 & 0.23 & 0.25 & 0.44 & 0.39 \\
\hline 22 & 0.29 & 0.36 & 0.35 & 0.32 & 0.43 & 0.35 \\
\hline 23 & 0.11 & 0.14 & 0.16 & 0.16 & 0.48 & 1.00 \\
\hline 24 & 0.16 & 0.16 & 0.16 & 0.16 & 0.17 & 0.43 \\
\hline 25 & 0.35 & 0.41 & 0.43 & 0.45 & $0.01 \mathrm{sig}$ & 0.99 \\
\hline 26 & 0.19 & 0.21 & 0.22 & 0.21 & 0.90 & 0.06 \\
\hline
\end{tabular}

Table 4. Probability to remember one more word in 1, 2, 4 and 6 months in the treatment group

\begin{tabular}{|c|c|c|c|c|c|c|}
\hline Patient ID & $\begin{array}{l}\text { Prob to } \\
\text { remember one extra } \\
\text { word in } 1 \text { month }\end{array}$ & $\begin{array}{l}\text { Prob to } \\
\text { remember one extra } \\
\text { word in } 2 \text { months }\end{array}$ & $\begin{array}{l}\text { Prob to } \\
\text { remember one extra } \\
\text { word in } 4 \text { months }\end{array}$ & $\begin{array}{l}\text { Prob to } \\
\text { remember one extra } \\
\text { word in } 6 \text { months }\end{array}$ & $\begin{array}{l}\text { p-value for } \\
\mathrm{H}_{0}: 1+\phi=1\end{array}$ & $\begin{array}{l}\text { Power with } \\
\mathrm{H}_{1}: 1+\phi=2\end{array}$ \\
\hline 1 & 0.61 & 0.68 & 0.68 & 0.71 & 0.12 & 0.74 \\
\hline 2 & 0.42 & 0.47 & 0.53 & 0.61 & 0.04 sig & 1.00 \\
\hline 3 & 0.89 & 0.87 & 0.93 & 0.91 & 0.19 & 0.50 \\
\hline 4 & 0.59 & 0.58 & 0.56 & 0.54 & 0.82 & 0.08 \\
\hline 5 & 0.42 & 0.37 & 0.36 & 0.34 & 0.56 & 0.23 \\
\hline 6 & 0.67 & 0.56 & 0.57 & 0.60 & 0.52 & 0.20 \\
\hline 7 & 0.53 & 0.58 & 0.56 & 0.52 & 0.44 & 0.39 \\
\hline 8 & 0.77 & 0.72 & 0.76 & 0.77 & $0.01 \mathrm{sig}$ & 1.00 \\
\hline 9 & 0.20 & 0.20 & 0.17 & 0.18 & 0.68 & 0.19 \\
\hline 10 & 0.45 & 0.49 & 0.61 & 0.61 & $0.02 \mathrm{sig}$ & 1.00 \\
\hline 11 & 0.64 & 0.54 & 0.63 & 0.63 & 0.67 & 0.14 \\
\hline 12 & 0.14 & 0.14 & 0.14 & 0.18 & 1.00 & 1.00 \\
\hline 13 & 0.42 & 0.43 & 0.42 & 0.43 & 0.68 & 0.22 \\
\hline 14 & 0.10 & 0.12 & 0.09 & 0.07 & $\begin{array}{l}\text { outlier } \\
\text { case }\end{array}$ & $\begin{array}{l}\text { outlier } \\
\text { case }\end{array}$ \\
\hline 15 & 1.00 & 0.98 & 0.97 & 1.00 & 0.93 & 0.02 \\
\hline 16 & 0.87 & 0.87 & 0.86 & 0.83 & 0.72 & 0.10 \\
\hline 17 & 0.74 & 0.67 & 0.65 & 0.63 & $0.06 \mathrm{sig}$ & 0.41 \\
\hline 18 & 0.39 & 0.37 & 0.36 & 0.39 & 1.00 & 0.00 \\
\hline 19 & 0.61 & 0.54 & 0.56 & 0.56 & 0.58 & 0.18 \\
\hline
\end{tabular}


Scanning the probability for an Alzheimer's patient to remember just one-extra word in the list is computed in the control group in Table 3, we notice that all patients except those with ID \# 1, \# 8 have lesser than $50 \%$ chance. From the p-value for the null hypothesis $\mathrm{H}_{0}: 1+\phi^{8}=1$ to be according an Alzheimer's data in the end of 6 months in Table 3 for the control group, we notice that the null hypothesis is false only for patients with ID \# 4, \# 9, \# 10, \# 13, \# 15, \# 17, \# 18 and \# 25. Could the incidence rate have doubled in the control group? This amounts to considering $\phi^{*}=1$ in the amplifying factor. Scanning the power of accepting the true hypothesis $\mathrm{H}_{1}: 1+\phi^{8}=2$ with an Alzheimer's data in the end of 6 months in Table 3 , we notice that the power is good in many cases. The ROC curve of the Alzheimer's patients in the control group is sketched in Fig. 6 and it has a significant convexity suggesting that the methodology works well in the control group.

\subsection{Alzheimer's Patients in Treatment Group}

The patients in the treatment group are received lecithin drug. Scanning the estimates of the amplyfying factor in Table 2, we notice that only patients with ID \# 5, \# 9, \# 12, \# 13, \# 14, \# 18, \# 20 and \# 21 showed no improvement. The patient with ID \# 10 showed improvement after 2 months time. Other Alzheimer's patients in the treatment group showed improvement. The patient with ID \# 14 is an outlier case because of zero entries. Scanning the probability for an Alzheimer's patient to remember just one-extra word in the list is computed in the treatment group in Table 4, we notice that all patients except those with ID \# 5, \# 9, \# 12, \# 13, \# 18 and \# 20 have more than $50 \%$ chance. From the p-value for the null hypothesis $\mathrm{H}_{0}: 1+\phi^{8}=1$ to be according an Alzheimer's data in the end of 6 months in Table 3 for the control group, we notice that the null hypothesis is false only for patients with ID \# 2, \# 8, \# 10, \# 17, \# 21 and \# 22 . Could the incidence rate have doubled in the treatment group? This amounts to considering $\phi^{*}=1$ in the amplifying factor. Scanning the power of accepting the true hypothesis $\mathrm{H}_{1}: 1+\phi^{8}=2$ with an Alzheimer's data in the end of 6 months in Table 4, we notice that the power is good in many cases. The ROC curve of the Alzheimer's patients in the control and in the treatment group are sketched respectively in Fig. 6 and in Fig. 7. The configurations have significant convexity suggesting that the methodology works well in the control and the treatment groups.

\section{CONCLUSION}

There is no known cure of Alzheimer's disease. The Alzheimer's disease is fatal. Many clinical trials are performed by the research institutions, national labs and pharmaceutical companies. The human genome might be a great help to identify the gene marker for this disease. For a breakthrough to treat successfully, the data analysis by a suitable statistical methodology is a necessity. It is understandable that the difference in the performance of the Alzheimer's patients differs across the group. Why do some Alzheimer's patients differ from others in the same group? An answer to this question would open the door for new knowledge about the Alzheimer's disease. More pertinent data need to be collected. An analysis of such data using a regression methodology with covariates could well explain. More research work is anticipated to develop a suitable regression methodology for such data.

\section{REFERENCES}

Bapat, R.B., S.J. Kirkland, K.M. Prasad and S. Puntanen, 2013. Combinatorial Matrix Theory and Generalized Inverses of Matrices. 1st Edn., Springer London, Limited, New Delhi, ISBN-10: 8132210522, pp: 277.

Braak, H. and K.D. Tredici, 2012. Where, when and in what form does sporadic Alzheimer's disease begin?. Curr. Opinion Neurol., 25: 708-714. DOI: DOI: $10.1097 /$ WCO.0b013e32835a3432

Everitt, B.S. and A.R. Pickles, 2004. Statistical Aspects Of the Design and Analysis of Clinical Trials. 1st Edn., Imperiall College Press, London, ISBN-10: 1860945449, pp: 323.

Moore, E.A. and L. Moore, 2012. Encyclopedia of Alzheimer's Disease: with Directories of Research, Treatment and Care Facilities. 2nd Edn., McFarland and Company Incorporated Pub, Jefferson, N.C., pp: ISBN-10: 0786464585, pp: 447.

Stuart, A. and K. Ord, 1994. kendall's Advanced Theory of Statistics. 6st Edn., Wiley, London Griffin, ISBN-10: 0340614307, pp: 700. 